\title{
INERTIAL CONFINEMENT FUSION
}

\section{Fast Ignitor Concept with Light Ions}

\author{
V. Yu. Bychenkov ${ }^{1,2}$, W. Rozmus ${ }^{2}$, A. Maksimchuk ${ }^{3}$, D. Umstadter ${ }^{3}$, and C. E. Capjack ${ }^{4}$ \\ ${ }^{1}$ Lebedev Institute of Physics, Russian Academy of Sciences, Leninskiu pr. 53, Moscow, 119991 Russia \\ ${ }^{2}$ Theoretical Physics Institute, Department of Physics, University of Alberta, Edmonton T6G 2J1, Alberta, Canada \\ ${ }^{3}$ Center for Ultrafast Optical Science, University of Michigan, Ann Arbor, MI 48109-2099, USA \\ ${ }^{4}$ Department of Computer and Electrical Engineering, University of Alberta, Edmonton, Alberta, T6G 2J1, Canada
}

Received June 25, 2001

\begin{abstract}
A short-laser-pulse driven ion flux is examined as a fast ignitor candidate for inertial confinement fusion. Ion ranges in a hot precompressed fuel are studied. The ion energy and the corresponding intensity of a short laser pulse are estimated for the optimum ion range and ion energy density flux. It is shown that a lightion beam triggered by a few-hundreds-kJ laser at intensities of $\geq 10^{21} \mathrm{~W} / \mathrm{cm}^{2}$ is relevant to the fast ignitor scenario. () 2001 MAIK "Nauka/Interperiodica".
\end{abstract}

In the fast ignitor scenario [1], which is a milestone of the concept of inertial confinement fusion (ICF), a relativistic electron beam is considered to be the most suitable source for igniting a hot spot much smaller than the dense compressed DT core. Studies of the feasibility of fast ignition with relativistic electrons are now being carried out at many laboratories [2-5]. In addition, a $15-\mathrm{GeV}$ bismuth ion beam from an external source instead of an electron beam generated directly in the target corona was also examined [6].

Over the past year, there have been several observations of multi-MeV ion beams generated by high-intensity ultrashort laser pulses in the interaction with solid targets [5, 7-9]. In this context, the present paper aims to provide insight into the feasibility of the fast ignition concept with high energy beams of light ions generated in laser-plasma interaction. Apart from the standard studies about the electron fast ignitor concept for ICF, our main concern is to prove that a light-ion beam is capable of igniting a hot spot on a reasonable laser energy scale. In contrast to relativistic electron beams, ions are much less influenced by collective plasma phenomena and have straight-line trajectories. Light ions, similar to electrons, can be generated due to laserplasma interaction in a target, while a heavy ion beam must be produced by an external driver and transported to the target. Ion transport is not inhibited so much by the self-consistent electric field because the ions accelerated by the charge separation field at a near-critical density are much heavier and propagate inertially inside the target together with the electrons as a chargecompensated neutral beam. Below, the optimum parameters of an ion beam and laser pulse that are suitable for an ignition spark in a hot precompressed DT fuel are estimated as a rough guide.

The mechanism for ion acceleration is charge separation in a plasma due to high-energy electrons driven by the laser inside the target [9] and/or an inductive electric field as a result of the self-generated magnetic field [10]. These electrons can be accelerated up to multi-MeV energies due to several processes, such as stimulated Raman scattering [11], resonant absorption [12], laser wakefield [13], ponderomotive acceleration by standing [14] and propagating [15] laser pulses, "vacuum heating" due to the $\mathbf{V} \times \mathbf{B}$ Lorentz force [16] or Brunel effect [17], and betatron resonance provided by laser pulse channeling [18].

It is unlikely that the ponderomotive mechanism [14] at laser intensities higher than $10^{18} \mathrm{~W} / \mathrm{cm}^{2}$ can produce ions with the observed energies (see [9]). The maximum proton energy in experiments with foils at a high-contrast intensity ratio was explained by acceleration in the charge-separation field arising due to "vacuum heating" [9]. However, for the fast ignitor scheme, this mechanism is inapplicable because of the extended plasma corona at the front of the dense target. Recent experiments carried out at the Center for Ultrafast Optical Science [19] demonstrated a significant increase in the ion energy (as compared to [9]) if the laser intensity contrast ratio decreases. Thus, one may identify a preformed plasma as a source of enhanced electron generation and, hence, enhanced electrostatic field that efficiently accelerates the ions. We believe that the Raman scattering mechanism for electron forward acceleration [11] together with the laser channeling effects [18] are the most likely processes at the corona of an ICF target which produce a strong sheath electrostatic field and are responsible for ion beam generation by short laser pulses at laser intensities of $>10^{18} \mathrm{~W} / \mathrm{cm}^{2}$.

Hot electrons, accelerated in an underdense plasma (with a density $n_{e}$ comparable to the critical density $n_{c}$ ) up to the energy $\epsilon_{e}$, penetrate into the target at a distance on the order of the Debye length $\lambda_{D e} \propto \sqrt{\epsilon_{e} / n_{e}}$ and create a strong sheath electrostatic field, which accelerates ions forward. Acceleration gradients of sev- 
eral tens of $\mathrm{GeV} / \mathrm{cm}$ are expected for $\mathrm{MeV}$ electrons. As electrons are decelerated, their kinetic energy transforms into the electrostatic field energy and the electric potential should be expected to be at the level of the hot electron energy $\epsilon_{e}$. Correspondingly, the magnitude of the electric potential determines the ion energy $\epsilon \sim$ $Z e \phi \sim Z \epsilon_{e}$, where $e$ and $Z e$ are the electron and ion charges. Most of the measurements suggest that protons and deuterons are the major species of laser triggered particle emission, although heavy ions have recently been identified [20]. Their energy is proportional the charge number $Z$, which is consistent with the electrostatic process of ion acceleration. Clearly, an evaluation of the feasibility of fast ignition with energetic ions must be based on the conversion efficiency of the laser light into ion beam energy and scaling of the beam parameters versus laser characteristics. A systematic investigation of both of these issues has only begun. However, the data on the $\sim 6 \%$ conversion efficiency into ions of several $\mathrm{MeV}$ energy [8] and the square root dependence of the proton energy on the laser intensity inferred from the latest experiments are very promising.

The general approach to fast ignition involves a powerful external unspecified source and aims to define the ignition parameters for a beam and a core. The first study of fast ignitor parameters was presented by Tabak et al. [1]. As was pointed out in [21], the original fast ignitor concept [1] dealt with a nearly isobaric fuel configuration and underestimated the energy required for ignition, which is more relevant to a nearly isochoric process and is somewhat larger than first proposed. The results of [21] roughly agree with those presented in [6] and predict a larger ignition energy than that given by the analytical model of Piriz and Sanchez [22]. According to [21], the optimum particle range is $R=0.6 \mathrm{~g} / \mathrm{cm}^{2}$, while the model [22] predicts $R=0.25 \mathrm{~g} / \mathrm{cm}^{2}$. Regardless of the differences between [21] and [22], we consider a wide enough domain of the particle ranges to include both of these estimations.

Similar to [23], where the physics of the electron fast ignitor was discussed, our key issue includes an estimation of the ion penetration depth into the dense compressed DT core with a density of $\sim 300 \mathrm{~g} / \mathrm{cm}^{3}$ and temperature of $\sim 10 \mathrm{keV}$. For ions with energies higher than one-hundred $\mathrm{keV}$, the penetration depth is determined by their collisions with electrons; i.e., fast ions heat electrons of the core and lose energy in accordance with the equation [24]

$$
\begin{gathered}
-\frac{d \epsilon}{d x}=\frac{2 \pi e^{4} Z^{2} n_{e}}{u T_{e}} \Lambda F(u), \\
F(u)=\psi(u)-\left(m_{e} / m_{i}\right) \psi^{\prime}(u),
\end{gathered}
$$

where $\epsilon$ is the ion energy, $T_{e}$ is the electron temperature, $m_{e(i)}$ is the mass of an electron (ion), $\Lambda$ is the Coulomb logarithm, $u=m_{e} \epsilon / m_{i} T_{e}$, and

$$
\psi(u)=\frac{2}{\sqrt{\pi}} \int_{0}^{u} d t \sqrt{t} e^{-t} .
$$

The Coulomb logarithm in Eq. (1),

$$
\Lambda \simeq 33-\ln \left[Z \sqrt{n_{e}\left(1+Z T_{e} / \epsilon\right)} / T_{e}^{3 / 2}(1+u)\right],
$$

where the electron density and temperature are in $\mathrm{cm}^{-3}$ and $\mathrm{keV}$, respectively, depends only slightly on the ion energy for the parameters of particular interest $\left(Z m_{e} / m_{i} \lesssim u \lesssim 1\right)$ and typically is $6-8$.

We define the ion range $R$ (in $\mathrm{g} / \mathrm{cm}^{2}$ ) as where a particle loses its energy up to the double thermal plasma energy $\left(3 T_{e}\right)$ :

$$
\begin{gathered}
R=0.06 \frac{A T_{e}^{2}}{Z^{2}} \int_{u_{0}}^{u} \frac{u d u}{\Lambda F}, \\
\Lambda=\frac{1}{2}\left[13-\ln \left(\frac{\rho\left(Z^{2}+5.5 \times 10^{-4} Z^{3} / A u\right)}{(1+u)^{2} T_{e}^{3}}\right)\right],
\end{gathered}
$$

where $A$ is the atomic number of a projectile and $u_{0}=$ $3 m_{e} / m_{i}$. In Eq. (3) and below, $\epsilon$ is in $\mathrm{MeV}, T_{e}$ is in $\mathrm{keV}$, $\rho$ is in $\mathrm{g} / \mathrm{cm}^{3}$, and $R$ is in $\mathrm{g} / \mathrm{cm}^{2}$. For moderately energetic ions $\left(0.01 Z T_{e} \ll \epsilon \ll A T_{e}\right)$, one can estimate range (3) and present $R$ in a simple form

$$
R=\frac{0.08}{\Lambda Z^{2}} \sqrt{A T_{e}^{3} \epsilon}
$$

where $\Lambda \simeq 6.5-\ln \left(Z \sqrt{\rho} / T_{e}^{3 / 2}\right)$. In Fig. 1, range (3) for $\mathrm{H}, \mathrm{D}$, and Be ions is shown versus the energy $\epsilon$ in a core with the temperature $T_{e}=5,10$, and $15 \mathrm{keV}$ and density $\rho=300 \mathrm{~g} / \mathrm{cm}^{3}$. The domain between the dashed lines in Fig. 1 corresponds to the region $0.15<R<1.2 \mathrm{~g} / \mathrm{cm}^{2}$, where, according to [21], the ignition parameters were found to depend very little on $R$. We assume below that this domain of parameters is required for the ignition of a hot spot. Note that Eq. (4) qualitatively describes the dependence of the particle range on the ion energy and plasma parameters and, for the examples given in Fig. 1, estimates $R$ with an accuracy of $\sim 50 \%$, somewhat underestimating the particle range. Figure 1 shows that a significantly higher energy is required for Be ions as compared to protons and deuterons.

In accordance with [21, 22], the minimum intensity of the pulse of fast particles $I_{p}$ required for ignition is slightly less than $10^{20} \mathrm{~W} / \mathrm{cm}^{2}$. We accept as a rough guide $I_{p}$ as given in [21] $\left(I_{p} \simeq 6.5 \times 10^{19} \mathrm{~W} / \mathrm{cm}^{2}\right)$ to examine whether the required ion energy is consistent with the ignition window for $R$ shown in Fig. 1 . We estimate the typical ion density $n_{i}$ from the quasineutrality condition $n_{i} \approx n_{e} / Z$, choosing a hot electron density $n_{e}$ equal to approximately the quarter critical value $n_{c} / 4$, 
typical of the Raman instability, which efficiently accelerates electrons in the forward direction. The critical density reads as $n_{c} \simeq 0.85 \times 10^{21} \lambda^{-1} \sqrt{I / 10^{18}} \mathrm{~cm}^{-3}$, where the laser wavelength $\lambda$ and intensity $I$ are in $\mu \mathrm{m}$ and $\mathrm{W} / \mathrm{cm}^{2}$, respectively, and the plasma relativistic transparency is taken into account.

Apparently, no theory exists that describes the ion energy dependence on the laser intensity. However, some recent experiments $[8,9,20]$ have shed light on this issue. Figure 2 shows that, at the intensities $5 \times 10^{18} \leq I \leq 3 \times 10^{20} \mathrm{~W} / \mathrm{cm}^{2}$ of $1-\mu \mathrm{m}$ laser light, the maximum proton energy $\epsilon_{\max }$ is well approximated by a square root dependence $\epsilon_{\max } \simeq 3.6 \sqrt{I / 10^{18}} \mathrm{MeV}$. However, the more relevant average energy of protons in the beam is several times smaller. We estimate it as $\epsilon_{\max } / 7$ in accordance with the results of [9], where the effective temperature and maximum energy of the protons was $230 \mathrm{keV}$ and $1.5 \mathrm{MeV}$, respectively. Such an estimate also agrees with the results of [8]. Therefore, the scaling

$$
\epsilon \approx 0.5 Z \lambda \sqrt{I / 10^{18}} \mathrm{MeV}
$$

can be inferred from recent experiments on high energy ion generation. Note that the Z-proportionality in Eq. (5) corresponds to the electrostatic mechanism for ion acceleration. However, Eq. (5) may overestimate the ion energy with a specific charge number $Z$ in a multispecies plasma that contains lighter ions (with charge numbers smaller than $Z$ ). Heavier ions have little chance to be significantly accelerated because of their lower mobility. They follow behind the light-ion bunch and, therefore, experience a significantly lower accelerating electric field. Consequently, their energy should be significantly lower than the energy of light ions. This is why high-energy protons were mostly observed in the current experiments.

Using Eq. (5) for the estimation of the ion energy flux $I_{i} \simeq n_{i}\left(2 / m_{i}\right)^{1 / 2} \epsilon^{3 / 2}$, one can obtain

$$
I_{i} \approx 1.7 \times 10^{-2}\left(\frac{I}{10^{18}}\right)^{5 / 4} \sqrt{\frac{Z \lambda}{A}} \frac{\mathrm{W}}{\mathrm{cm}^{2}},
$$

where we assume that ions are nonrelativistic. Equation (6) predicts that a conversion efficiency of laser energy into energetic ions scales with an intensity of $I^{1 / 4}$ and, for $Z=1, \lambda=1 \mu \mathrm{m}$, and $I=3 \times 10^{20} \mathrm{~W} / \mathrm{cm}^{2}$, gives $7 \%$ for the conversion coefficient, which agrees well with the experiment [8].

From the condition $I_{i}=I_{p}$, we obtain the threshold intensity for a $1-\mu \mathrm{m}$ laser, which is found to be practically the same for proton $\left(1.2 \times 10^{21} \mathrm{~W} / \mathrm{cm}^{2}\right)$, deuteron $\left(1.5 \times 10^{21} \mathrm{~W} / \mathrm{cm}^{2}\right)$, and beryllium $\left(1.6 \times 10^{21} \mathrm{~W} / \mathrm{cm}^{2}\right)$ beams with typical ion energies of $6.8,7.7$, and $32 \mathrm{MeV}$, respectively. It is likely that the core temperature should be $T_{e} \geqslant 10 \mathrm{keV}$ (rather than $T_{e}<10 \mathrm{keV}$ ), which basically excludes deuterons as a possible ignitor
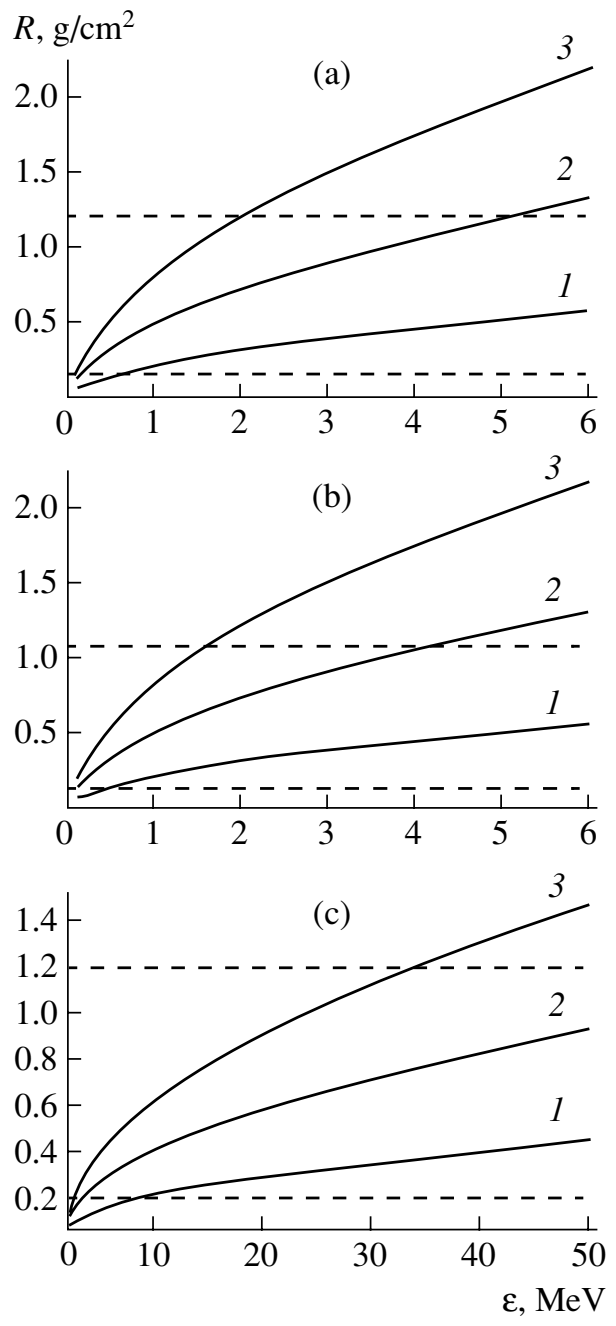

Fig. 1. The $R(\epsilon)$ dependence for (a) H, (b) D, and (c) Be ions for the core temperatures (1) 5, (2) 10 , and (3) $15 \mathrm{keV}$.

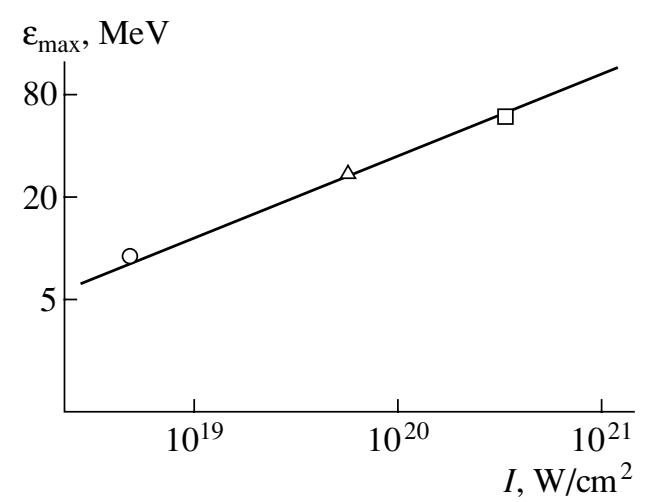

Fig. 2. Maximum proton energy vs. the laser intensity. The square, circle, and triangle mark the results of $[8,9,20]$, respectively. The solid line is the fit $\epsilon_{\max } \simeq 3.6 \sqrt{I / 10^{18}} \mathrm{MeV}$, where $I$ is in $\mathrm{W} / \mathrm{cm}^{2}$. 
because its range is too long (Fig. 1b) for this temperature. Such a situation is somewhat similar to that with a proton beam. However, it has a chance to be used as an ignitor at the upper boundary of the range ignition window, $R \simeq 1.2 \mathrm{~g} / \mathrm{cm}^{2}$ (Fig. 1a). The energy $\epsilon \simeq 32 \mathrm{MeV}$ of a beryllium ion beam for a core temperature of $T_{e} \sim$ $10 \mathrm{keV}$ agrees well with $R \approx 0.6 \mathrm{~g} / \mathrm{cm}^{2}$, which was interpreted in [21] as the optimum for ignition. We note that deuterons were discarded because of their long range, as compared to $R=1.2 \mathrm{~g} / \mathrm{cm}^{2}$ found in [21] as an upper limit for fast ignition. The latter should be reconsidered if a new optimum regime for ignition is found for longer ion ranges. In addition, deuterons with energies of several $\mathrm{MeV}$ propagating in a dense DT plasma may participate in thermonuclear reactions. However, our estimates show that all the possible nuclear channels of energy release cannot provide more than several percent of the additional energy, which does not affect our conclusion.

As a final remark, we note that one of the key issues of fast ignition is the self-heating of a hot spot. This cannot be achieved in a hot spot that is too small, so the penetration depth cannot be shorter than the hot-spot diameter and, consequently, the ignition energy should be large enough. However, analytical model [22] and numerical hydrodynamic simulations [21] give different estimates for the hot-spot size. The latter predicted a much higher energy threshold for the triggering of spark ignition, which is $\simeq 17 \mathrm{~kJ}$ for the optimum set of parameters. Accepting this more pessimistic estimate, we conclude that ignition with a light-ion beam might be expected at a $\gtrsim 200-\mathrm{kJ}$ laser energy.

In summary, we have examined the feasibility of light-ion triggered spark ignition for ICF using the computation of the ion range in a hot core and recent experimental results on high-energy ion generation in laser-plasma interactions. The estimated requirements for short laser pulses correspond to an intensity of $\geqslant 10^{21} \mathrm{~W} / \mathrm{cm}^{2}$ and energy $\gtrsim 200 \mathrm{~kJ}$. The weak dependence of the required laser intensity on the ion atomic number has been found from a comparison of proton, deuteron, and beryllium ion beams. This may have practical importance, making fast ignition quite insensitive to the ion species.

Originally, this work was prepared for the journal Comments on Plasma Physics and Controlled Fusion, where it was accepted in autumn 2000 for publication in the first issue for 2001. Because of the change of the owner and his wish to stop the publishing of Comments on Plasma Physics and Controlled Fusion, the manuscript was resubmitted for Plasma Physics Reports. At that time, we were aware of the publication of paper [25] devoted to fast ignition with a proton beam.

\section{ACKNOWLEDGMENTS}

This work was supported in part by the Russian Foundation for Basic Research (project no. 00-02-
16063), the Natural Sciences and Engineering Research Council of Canada, and the National Science Foundation of USA.

\section{REFERENCES}

1. M. Tabak, I. Hammer, E. M. Glinsky, et al., Phys. Plasmas 1, 1626 (1994).

2. M. H. Key, M. D. Cable, T. E. Cowan, et al., Phys. Plasmas 5, 1966 (1998).

3. T. A. Hall, S. Ellwi, D. Batani, et al., Phys. Rev. Lett. 81, 1003 (1998).

4. L. Gremillet, F. Amiranoff, S. D. Baton, et al., Phys. Rev. Lett. 83, 5015 (1999).

5. K. A. Tanaka, R. Kodama, H. Fujita, et al., Phys. Plasmas 7, 2014 (2000).

6. A. Caruso and V. A. Pais, Nucl. Fusion 36, 745 (1996); Phys. Lett. A 243, 319 (1998).

7. E. L. Clark, K. Krushelnick, J. R. Davies, et al., Phys. Rev. Lett. 84, 670 (2000).

8. S. P. Hatchett, C. G. Brown, T. E. Cowan, et al., Phys. Plasmas 7, 2076 (2000).

9. A. Maksimchuk, S. Gu, K. Flippo, et al., Phys. Rev. Lett. 84, 4108 (2000).

10. T. Zh. Esirkepov, Y. Sentoku, K. Mima, et al., Pis'ma Zh. Éksp. Teor. Fiz. 70, 80 (1999) [JETP Lett. 70, 82 (1999)].

11. C. D. Decker, W. B. Mori, and T. Katsouleas, Phys. Rev. E 50, 3338 (1994).

12. S. C. Wilks and W. L. Kruer, IEEE J. Quantum Electron. 33, 1954 (1997).

13. T. Tajima and J. M. Dawson, Phys. Rev. Lett. 43, 267 (1979).

14. S. C. Wilks, W. L. Kruer, M. Tabak, and A. B. Langdon, Phys. Rev. Lett. 69, 1383 (1992).

15. W. Yu, V. Bychenkov, Y. Sentoku, et al., Phys. Rev. Lett. 85, 570 (2000).

16. W. L. Kruer and K. Estabrook, Phys. Fluids 28, 430 (1985).

17. F. Brunel, Phys. Rev. Lett. 59, 52 (1987).

18. A. Pukhov, Z.-M. Sheng, and J. Meyer-ter-Vehn, Phys. Plasmas 6, 2847 (1999).

19. K. Nemoto, S. Banerjee, K. Flippo, et al., Appl. Phys. Lett. 78, 595 (2001).

20. K. Krushelnick, E. L. Clark, M. Zepf, et al., Phys. Plasmas 7, 2055 (2000).

21. S. Atzeni, Jpn. J. Appl. Phys. 34, 1980 (1995); Phys. Plasmas 6, 3316 (1999).

22. A. R. Piriz and M. M. Sanchez, Phys. Plasmas 5, 2721 (1998); 5, 4373 (1998).

23. C. Deutsch, H. Furukawa, K. Mima, et al., Phys. Rev. Lett. 77, 2483 (1996).

24. B. A. Trubnikov, in Reviews of Plasma Physics, Ed. by M. A. Leontovich (Gosatomizdat, Moscow, 1963; Consultants Bureau, New York, 1963), Vol. 1.

25. M. Roth, T. E. Cowan, M. H. Key, et al., Phys. Rev. Lett. 86, 436 (2001).

Translated by the authors

PLASMA PHYSICS REPORTS Vol. $27 \quad$ No. $12 \quad 2001$ 